\title{
FIXED POINTS ON TRANSVERSAL EDGES SPACES
}

\author{
Milan R. Tasković ${ }^{*}$
}

\begin{abstract}
In this paper we formulate a new structure of spaces which we call it edges (upper or lower) transversal spaces. Also, in this sense, we describe a class of conditions sufficient for the existence of a fixed point on edges (upper or lower) transversal spaces.
\end{abstract}

\section{Introduction and history}

The possibility of defining such notions as limit and continuity in an arbitrary set is an idea which undoubtedly was first put forward by M. Fréchet in 1904, and developed by him in his famous doctoral dissertation 1905.

In this paper we introduce a new concept by name edges transversal spaces as a nature extension of Fréchet's spaces. In the second part, of this paper, applications in the fixed point theory are considered.

In this sense, first, let $(X, \rho)$ be a metric space and $T$ a mapping of $X$ into itself. A metric space $X$ is said to be T-orbitally complete iff every Cauchy sequence which is contained in orbit $\mathcal{O}(x)=\left\{x, T x, T^{2} x, \ldots\right\}$ for some $x \in X$ converges in $X$.

In 1980 I have been proved the following result of fixed point on metric spaces which has a best long of all known sufficiently conditions for the existing of unique fixed point, cf. Tasković [2], [3] and [5]. This result generalizes a great number of known results.

AMS (MOS) Subject Classification 1991. Primary: 54E15, 47H10, 05A15. Secondary: 54E35, 54H25.

Key words and phrases: Transversal spaces, Edges (upper or lower) transversal spaces, Fréchet's spaces, Fixed point theorems, diametral $\varphi$-contraction, upper (or lower) edges contraction, edges (upper or lower) continuous, method of successive approximations.

*Research supported by Science Fund of Serbia under Grant 1457. 
In [2] I have been introduced the concept of a diametral $\varphi$-contraction $T$ of a metric space $(X, \rho)$ into itself, i.e., there exists a function $\varphi: \mathbb{R}_{+}^{0} \rightarrow$ $\mathbb{R}_{+}^{0}:=[0,+\infty)$ satisfying

$$
\left(\forall t \in \mathbb{R}_{+}:=(0,+\infty)\right)\left(\varphi(t)<t \quad \text { and } \quad \limsup _{z \rightarrow t+0} \varphi(z)<t\right)
$$

such that

$$
\rho[T x, T y] \leq \varphi\left(\operatorname{diam}\left\{x, y, T x, T y, T^{2} x, T^{2} y, \ldots\right\}\right)
$$

for all $x, y \in X$, where diam denoted diameter.

In [2] Tasković has proved the following result: Let $T$ be a diametral $\varphi$ contraction on a T-orbitally complete metric space $(X, \rho)$. If diam $\mathcal{O}(x) \in \mathbb{R}_{+}^{0}$, then $T$ has a unique fixed point $\xi \in X$.

A brief first proof of this statement may be found in Tasković [2]. Also some brief proofs for this we can see in Tasković [3], [4] and [5]. For some results in connection with this see Ohta-Nikaido [9].

\section{Upper edges spaces}

Let $X$ be a nonempty set. The function $\rho: X \times X \rightarrow[a, b]$ for $a<b$ $\left(a, b \in \mathbb{R}_{+}^{0}:=[0,+\infty)\right)$ is called an upper edges transverse on $X$ (or upper transversal) iff: $\rho[x, y]=\rho[y, x], \rho[x, y]=a$ if and only if $x=y$, and if there is a function $\psi:[a, b]^{2} \rightarrow[a, b]$ such that

$$
\rho[x, y] \leq \max \{\rho[x, z], \rho[z, y], \psi(\rho[x, z], \rho[z, y])\}
$$

for all $x, y, z \in X$.

An upper edges transversal space (or upper edges space) is a set $X$ together with a given upper edges transverse on $X$. The function $\psi:[a, b]^{2} \rightarrow$ $[a, b]$ in $(\mathrm{A})$ is called upper bisection function.

From (A) it follows by induction that there is a function $G:[a, b]^{n} \rightarrow$ $[a, b]$ for $a<b$ such that

$$
\begin{gathered}
\rho\left[x_{0}, x_{n}\right] \leq \\
\leq \max \left\{\rho\left[x_{0}, x_{1}\right], \ldots, \rho\left[x_{n-1}, x_{n}\right], G\left(\rho\left[x_{0}, x_{1}\right], \ldots, \rho\left[x_{n-1}, x_{n}\right]\right)\right\}
\end{gathered}
$$

for all $x_{0}, x_{1}, \ldots, x_{n} \in X$ and for arbitrary fixed integer $n \geq 2$.

Example 1. (Metric spaces). A fundamental first example of upper edges space is a metric space. Indeed, if $(X, d)$ is a metric space, then for the upper bisection function $\psi(r, t)=r+t$ we have the following upper edges transverse $\rho: X \times X \rightarrow$ $[a, b] \subset \mathbb{R}_{+}^{0}$ for $a<b$ defined by

$$
\rho[x, y]=\frac{(b-a) d(x, y)}{1+d(x, y)}+a
$$


for all $x, y \in X$. Thus $(X, \rho)$ is an example of an upper edges transversal space. In general, every metric space is an example of an upper edges transversal space.

Example 2. (The extended real line $\overline{\mathbb{R}}$ ). The function $f$ defined in $\mathbb{R}$ by $f(x)=x /(1+|x|)$ is a bijection on $\mathbb{R}$ on the open interval $(-1,1) \subset \mathbb{R}$, and the inverse mapping $g$ being defined by $g(x)=x /(1-|x|)$ for $|x|<1$. Let $\overline{\mathbb{R}}$ be the set which is the union of $\mathbb{R}$ and two new elements written $+\infty$ and $-\infty$ (points at infinity); then we extend $f$ to a bijection of $\overline{\mathbb{R}}$ onto $[-1,1]$ by putting $f(+\infty)=1$, $f(-\infty)=-1$, and write again $g$ for the inverse mapping.

We can apply this process described to define $\overline{\mathbb{R}}$ as an upper edges transversal space by putting for the upper edges transverse $\rho: \overline{\mathbb{R}} \times \overline{\mathbb{R}} \rightarrow[0,2]$ that is

$$
\rho[x, y]=\left|\frac{x}{1+|x|}-\frac{y}{1+|y|}\right|
$$

for all $x, y \in \overline{\mathbb{R}}$. (We notice that for $x \geq 0$ is $\rho[+\infty, x]=1 /(1+|x|)$, and for $x \leq 0$ that is $\rho[-\infty, x]=1 /(1+|x|))$.

For any nonempty set $Y$ in the upper edges transversal space $X$, the diameter of $Y$ is defined as

$$
\operatorname{diam}(Y):=\sup \{\rho[x, y]: x, y \in Y\}
$$

it is a real number in $[a, b], A \subset B \operatorname{implies} \operatorname{diam}(A) \leq \operatorname{diam}(B)$. The relation $\operatorname{diam}(Y)=a$ holds if and only if $Y$ is a one point set.

Elements of an upper edges transversal space will usually be called points. Given an upper edges transversal space $(X, \rho)$, with the bisection function $g:[a, b]^{2} \rightarrow[a, b]$ and a point $z \in X$, the open ball of center $z$ and radius $r>0$ is the set

$$
g(B(z, r))=\{x \in X: \rho[z, x]<a+r\} .
$$

The convergence $x_{n} \rightarrow x$ as $n \rightarrow \infty$ in the upper edges transversal space $(X, \rho)$ means that

$$
\rho\left[x_{n}, x\right] \rightarrow a \quad \text { as } \quad n \rightarrow \infty,
$$

or equivalently, for every $\varepsilon>0$ there exist on integer $n_{0}$ such that the relation $n \geq n_{0}$ implies $\rho\left[x_{n}, x\right]<a+\varepsilon$.

The sequence $\left\{x_{n}\right\}_{n \in \mathbb{N}}$ in the upper edges transversal space $(X, \rho)$ is called transversal sequence (or upper Cauchy sequence) iff for every $\varepsilon>0$ there is an $n_{0}=n_{0}(\varepsilon)$ such that

$$
\rho\left[x_{n}, x_{m}\right]<a+\varepsilon \text { for all } n, m \geq n_{0} .
$$

Let $(X, \rho)$ be an upper edges transversal space and $T: X \rightarrow X$. We notice, from Tasković [7], that a sequence of iterates $\left\{T^{n}(x)\right\}_{n \in \mathbb{N}}$ in $X$ is said to be transversal sequence if and only if

$$
\lim _{n \rightarrow \infty}\left(\operatorname{diam}\left\{T^{k}(x): k \geq n\right\}\right)=a .
$$


In this sense, an upper edges transversal space is called upper complete iff every transversal sequence converges. Also, a space $(X, \rho)$ is said to be upper orbitally complete (or upper T-orbitally complete) iff every transversal sequence which is contained in $\mathcal{O}(x):=\left\{x, T x, T^{2} x, \ldots\right\}$ for some $x \in X$ converges in $X$.

For further facts on upper edges transversal spaces see: Tasković [7].

A function $f$ mapping $X$ into the reals is $T$-orbitally lower semicontinuous at $p \in X$ if $\left\{x_{n}\right\}_{n \in \mathbb{N}}$ is a sequence in $\mathcal{O}(x)$ and $x_{n} \rightarrow p(n \rightarrow \infty)$ implies that $f(p) \leq \liminf f\left(x_{n}\right)$.

Let $(X, \rho)$ be an upper edges transversal space. A mapping $T: X \rightarrow X$ is said to be upper edges contraction if there exists an $0 \leq \lambda<1$ such that

$$
\rho[T(x), T(y)] \leq \lambda \rho[x, y]+a(1-\lambda)
$$

for all points $x, y \in X$.

In addition, let $\left(X, \rho_{X}\right)$ and $\left(Y, \rho_{Y}\right)$ be two upper edges transversal spaces and $T: X \rightarrow Y$. We notice, from Tasković [7], that $T$ be upper edges continuous at $x_{0} \in X$ iff for every $\varepsilon>0$ there exists a $\delta>0$ such that the relation

$$
\rho_{X}\left[x_{0}, x\right]<a+\delta \quad \text { implies } \rho_{Y}\left[T\left(x_{0}\right), T(x)\right]<a+\varepsilon .
$$

A typical first example of an upper edges continuous mapping is the upper edges contraction on the upper edges transversal space $(X, \rho)$. For the further facts on the upper edges continuous mappings see: Tasković [7].

We are now in a position to formulate the following statement, which is roofing for a great number of known result on metric spaces in the fixed point theory.

Theorem 2.1. Let $T$ be a mapping of an upper edges transversal space $(X, \rho)$ into itself and let $X$ be upper T-orbitally complete. Suppose that there exists a function $\varphi:[a, b] \rightarrow[a, b]$ satisfying

$$
(\forall t \in(a, b])\left(\varphi(t)<t \quad \text { and } \quad \limsup _{z \rightarrow t+0} \varphi(z)<t\right)
$$

such that

$$
\rho[T x, T y] \leq \varphi\left(\operatorname{diam}\left\{x, y, T x, T y, T^{2} x, T^{2} y, \ldots\right\}\right)
$$

for all $x, y \in X$. If $x \mapsto \operatorname{diam} \mathcal{O}(x)$ or $x \mapsto \rho[x, T x]$ is T-orbitally lower semicontinuous, then $T$ has a unique fixed point $\xi \in X$ and $\left\{T^{n}(x)\right\}_{n \in \mathbb{N}}$ converges to $\xi$ for every $x \in X$.

We begin the proof with the following lemma (as a well known lemma) which is essential in the following context. 
Lemma 1. (Tasković [7]). Let the mapping $\varphi:[a, b] \rightarrow[a, b] \subset \mathbb{R}_{+}^{0}$ for $a<b$ have the property (Iu). If the sequence $\left(x_{n}\right)$ of nonnegative real numbers satisfies the condition

$$
x_{n+1} \leq \varphi\left(x_{n}\right), \quad n \in \mathbb{N},
$$

then the sequence $\left(x_{n}\right)$ tends to $a$. The velocity of this convergence is not necessarily geometrical.

A brief first proof of this statement may be found in: Taskovic [7]. Other brief proofs for this we can see in Tasković [1], [2] and [5].

Proof of Theorem 1. Let $x$ be an arbitrary point in $X$. We can show then that the sequence of iterates $\left\{T^{n} x\right\}_{n \in \mathbb{N}}$ is a transversal sequence. It is easy to verify that the sequence $\left\{T^{n} x\right\}_{n \in \mathbb{N}}$ satisfies the following inequality

$$
\operatorname{diam} \mathcal{O}\left(T^{n+1}(x)\right) \leq \varphi\left(\operatorname{diam} \mathcal{O}\left(T^{n}(x)\right)\right.
$$

for $n \in \mathbb{N}$, and hence applying Lemma 1 to the sequence $\left(\operatorname{diam} \mathcal{O}\left(T^{n}(x)\right)\right.$ we obtain $\lim _{n \rightarrow \infty} \operatorname{diam} \mathcal{O}\left(T^{n}(x)\right)=a$. This implies that $\left\{T^{n}(x)\right\}_{n \in \mathbb{N}}$ is a transversal sequence in $X$ and, by upper $T$-orbitally completeness, there is a $\xi \in X$ such that $T^{n}(x) \rightarrow \xi(n \rightarrow \infty)$. Since $x \mapsto \operatorname{diam} \mathcal{O}(x)$ is $T$-orbitally lower semicontinuous at $\xi$,

$$
\rho[\xi, T \xi] \leq \operatorname{diam} \mathcal{O}(\xi) \leq \liminf _{n \rightarrow \infty}\left(\operatorname{diam} \mathcal{O}\left(T^{n}(x)\right)\right)=a ;
$$

thus $T \xi=\xi$, and we have shown that for each $x \in X$ the sequence $\left\{T^{n}(x)\right\}_{n \in \mathbb{N}}$ converges to a fixed point of $T$.

On the other hand, if $x \mapsto \rho[x, T x]$ is a $T$-orbitally lower semicontinuous at $\xi$, we have

$$
\rho[\xi, T \xi] \leq \liminf _{n \rightarrow \infty} \rho\left[T^{n} x, T^{n+1} x\right] \leq \liminf _{n \rightarrow \infty}\left(\operatorname{diam} \mathcal{O}\left(T^{n}(x)\right)=a ;\right.
$$

and thus again $T \xi=\xi$, i.e., we have again shown that for each $x \in X$ the sequence $\left\{T^{n}(x)\right\}_{n \in \mathbb{N}}$ converges to a fixed point of $T$.

We complete the proof by showing that $T$ can have at most one fixed point: for, if $\xi \neq \eta$ were two fixed points, then

$$
\begin{gathered}
a<\rho[\xi, \eta]=\rho[T \xi, T \eta] \leq \\
\leq \varphi\left(\operatorname{diam}\left\{\xi, \eta, T \xi, T \eta, T^{2} \xi, T^{2} \eta, \ldots\right\}\right)=\varphi(\operatorname{diam}\{\xi, \eta, \xi, \eta, \ldots\})<\rho[\xi, \eta],
\end{gathered}
$$

a contradiction. The proof is complete.

As immediate consequences of the preceding Theorem 1 we obtain directly the following interesting cases of $(\mathrm{B})$ :

(1) There exists a nondecreasing function $\varphi:[a, b] \rightarrow[a, b] \subset \mathbb{R}_{+}^{0}$ for $a<b$ satisfying $\lim \sup _{z \rightarrow t+0} \varphi(z)<t$ for every $t \in(a, b]$ such that

$$
\rho[T x, T y] \leq \varphi(\operatorname{diam}\{x, y, T x, T y\})
$$


for all $x, y \in X$.

(2) (Special affine case of condition (B) for $\varphi(t)=\alpha t+a(1-\alpha)$ ). There exists a constant $\alpha \in[0,1)$ such that for all $x, y \in X$ the following inequality holds

$$
\rho[T x, T y] \leq \alpha \operatorname{diam}\{x, y, T x, T y\}+a(1-\alpha),
$$

i.e., equivalently to

$$
\begin{gathered}
\rho[T x, T y] \leq \\
\leq \alpha \max \{\rho[x, y], \rho[x, T x], \rho[y, T y], \rho[x, T y], \rho[y, T x]\}+a(1-\alpha)
\end{gathered}
$$

(3) There exists a nondecreasing function $\varphi:[a, b] \rightarrow[a, b] \subset \mathbb{R}_{+}^{0}$ for $a<b$ satisfying $\lim \sup _{z \rightarrow t+0} \varphi(z)<t$ for every $t \in(a, b]$ such that

$$
\rho[T x, T y] \leq \varphi\left(\operatorname{diam}\left\{x, y, T x, T y, \ldots, T^{k} x, T^{k} y\right\}\right)
$$

for an arbitrary fixed integer $k \geq 0$ and for all $x, y \in X$.

(4) There exists an increasing mapping, i.e., $x_{i} \leq y_{i}(i=1, \ldots, 5)$ implies $f\left(x_{1}, \ldots, x_{5}\right) \leq f\left(y_{1}, \ldots, y_{5}\right), f:[a, b]^{5} \rightarrow[a, b] \subset \mathbb{R}_{+}^{0}$ for $a<b$ satisfying $\lim \sup _{z \rightarrow t+0} f(z, z, z, z, z)<t$ for every $t \in(a, b]$ such that

$$
\rho[T x, T y] \leq f(\rho[x, y], \rho[x, T x], \rho[y, T y], \rho[x, T y], \rho[y, T x])
$$

for all $x, y \in X$.

In connection with the preceding facts, we are now in a position to formulate a localization of Theorem 1 in the following form.

Theorem 2.2. Let $T$ be a mapping of an upper edges transversal space $(X, \rho)$ into itself and let $X$ be upper T-orbitally complete. Suppose that there exists a function $\varphi:[a, b] \rightarrow[a, b]$ satisfying (Iu) such that

$$
\operatorname{diam}\left\{T x, T^{2} x, \ldots\right\} \leq \varphi\left(\operatorname{diam}\left\{x, T x, T^{2} x, \ldots\right\}\right)
$$

for every $x \in X$. If $x \mapsto \operatorname{diam} \mathcal{O}(x)$ or $x \mapsto \rho[x, T x]$ is T-orbitally lower semicontinuous, then $T$ has a fixed point.

Proof of this statement is a totally analogous with the preceding proof of Theorem 1, and thus we omit it.

\section{Lower edges spaces}

Let $X$ be a nonempty set. The function $\rho: X \times X \rightarrow[a, b] \subset \mathbb{R}_{+}^{0}$ for $a<b$ is called a lower edges transverse on $X$ (or lower transversal) iff: 
$\rho[x, y]=\rho[y, x], \rho[x, y]=b$ if and only if $x=y$, and if there is a function $d:[a, b]^{2} \rightarrow[a, b]$ such that

$$
\rho[x, y] \geq \min \{\rho[x, z], \rho[z, y], d(\rho[x, z], \rho[z, y])\}
$$

for all $x, y, z \in X$.

A lower edges transversal space (or lower edges space) is a set $X$ together with a given lower edges transverse on $X$. The function $d:[a, b]^{2} \rightarrow$ $[a, b]$ in $(\mathrm{M})$ is called lower bisection function.

From (M) it follows by induction that there is a function $\mathcal{D}:[a, b]^{n} \rightarrow$ $[a, b]$ for $a<b$ such that

$$
\begin{gathered}
\rho\left[x_{0}, x_{n}\right] \geq \\
\geq \min \left\{\rho\left[x_{0}, x_{1}\right], \ldots, \rho\left[x_{n-1}, x_{n}\right], \mathcal{D}\left(\rho\left[x_{0}, x_{1}\right], \ldots, \rho\left[x_{n-1}, x_{n}\right]\right)\right\}
\end{gathered}
$$

for all $x_{0}, x_{1}, \ldots, x_{n} \in X$ and for arbitrary fixed integer $n \geq 2$.

Example 3. (Metric spaces). A fundamental first example of lower edges transversal space is a metric space. Indeed, if $(X, q)$ is a metric space, then for the lower bisection function $d(r, t)=r+t$ we have the following lower edges transverse $\rho: X \times X \rightarrow[a, b] \subset \mathbb{R}_{+}^{0}$ for $a<b$ defined by

$$
\rho[x, y]=\frac{(a-b) q[x, y]}{1+q[x, y]}+b
$$

for all $x, y \in X$. Thus $(X, \rho)$ is an example of a lower edges transversal space. In general, every metric space is an example of a lower edges transversal space.

Example 4. (Lower probabilistic spaces). A mapping $F: \mathbb{R} \rightarrow \mathbb{R}_{+}^{0}$ is called a distribution function if it is nondecreasing, left-continuous with inf $F=0$ and $\sup F=1$. We will denote by $\mathcal{L}$ the set of all distribution functions. We shall denote the distribution function $\mathcal{F}(p, q)$ by $F_{p, q}(x)$, whence $F_{p, q}(x)$ will denote the value of $F_{p, q}$ at $x \in \mathbb{R}$.

An example of lower edges transversal space is a lower probabilistic space which is a nonempty set $X$ together with the functions $F_{p, q}(x)$ with the following properties: $F_{p, q}(x)=F_{q, p}(x), F_{p, q}(0)=0$,

$$
F_{p, q}(x)=1 \text { for } x>0 \text { if and only if } p=q,
$$

and if there is a nondecreasing function $\tau:[0,1]^{2} \rightarrow[0,1]$ with property $\tau(t, t) \geq t$ for all $t \in[0,1]$ such that

$$
F_{p, q}(x+y) \geq \tau\left(F_{p, r}(x), F_{r, q}(y)\right)
$$

for all $p, q, r \in X$ and for all $x, y \geq 0$. If we chosen a lower bisection function $d:[0,1]^{2} \rightarrow[0,1]$ such that $d=\tau$ (from $(\mathrm{Nm})$ ), then we immediate obtain that every lower probabilistic space, for $\rho[p, q]=F_{p, q}(x): X \times X \rightarrow[0,1]$, is a lower edges transversal space. 
For any nonempty set $S$ in the lower edges transversal space $X$ the diameter of $S$ is defined as

$$
\operatorname{diam}(S):=\inf \{\rho[x, y]: x, y \in S\}
$$

it is a real number in $[a, b], A \subset B$ implies $\operatorname{diam}(B) \leq \operatorname{diam}(A)$. The relation $\operatorname{diam}(S)=b$ holds if and only if $S$ is a one point set.

Elements of a lower edges transversal space will usually be called points. Given a lower edges transversal space $(X, \rho)$, with the bisection function $d$ : $[a, b]^{2} \rightarrow[a, b]$ and a point $z \in X$, the open ball of center $z$ and radius $r>0$ is the set

$$
d(B(z, r))=\{x \in X: \rho[z, x]>b-r\} .
$$

The convergence $x_{n} \rightarrow x$ as $n \rightarrow \infty$ in the lower edges transversal space $(X, \rho)$ means that

$$
\rho\left[x_{n}, x\right] \rightarrow b \quad \text { as } n \rightarrow \infty,
$$

or equivalently, for every $\varepsilon>0$ there exist an integer $n_{0}$ such that the relation $n \geq n_{0}$ implies $\rho\left[x_{n}, x\right]>b-\varepsilon$.

The sequence $\left\{x_{n}\right\}_{n \in \mathbb{N}}$ in the lower edges transversal space $(X, \rho)$ is called transversal sequence (or lower Cauchy sequence) iff for every $\varepsilon>0$ there is an $n_{0}=n_{0}(\varepsilon)$ such that

$$
\rho\left[x_{n}, x_{m}\right]>b-\varepsilon \text { for all } n, m \geq n_{0} .
$$

Let $(X, \rho)$ be a lower edges transversal space and $T: X \rightarrow X$. We notice, from Tasković [7], that a sequence of iterates $\left\{T^{n}(x)\right\}_{n \in \mathbb{N}}$ in $X$ is said to be transversal sequence if and only if

$$
\lim _{n \rightarrow \infty}\left(\operatorname{diam}\left\{T^{k}(x): k \geq n\right\}\right)=b .
$$

In this sense, a lower edges transversal space is called lower complete iff every transversal sequence converges.

Also, a space $(X, \rho)$ is said to be lower orbitally complete (or lower $T$-orbitally complete) iff every transversal sequence which is contained in $\mathcal{O}(x)$ for some $x \in X$ converges in $X$.

A function $f$ mapping $X$ into the reals is $T$-orbitally upper semicontinuous at $p \in X$ iff $\left\{x_{n}\right\}_{n \in \mathbb{N}}$ is a sequence in $\mathcal{O}(x)$ and $x_{n} \rightarrow p(n \rightarrow \infty)$ implies that $f(p) \geq \lim \sup f\left(x_{n}\right)$.

Let $(X, \rho)$ be a lower edges transversal space. A mapping $T: X \rightarrow X$ is said to be lower edges contraction if there exists an $0 \leq \lambda<1$ such that

$$
\rho[T(x), T(y)] \geq \lambda \rho[x, y]+b(1-\lambda)
$$

for all points $x, y \in X$. For further facts on the lower edges contraction see: Tasković [7]. 
Theorem 3.3. (Method of successive approximations). Let $T$ be a mapping of a lower edges transversal space $(X, \rho)$ into itself and let $X$ be lower complete with an increasing bisection function d satisfying $d(t, t) \geq t$. If $T$ is a lower edges contraction, then:

(i) There exists one and only one $\xi \in X$ such that $T(\xi)=\xi$.

(ii) For any $x_{0} \in X$, the sequence of points

$$
x_{1}=T\left(x_{0}\right), \quad x_{2}=T\left(x_{1}\right), \quad x_{3}=T\left(x_{2}\right), \ldots
$$

tends to $\xi \in X$, and the following inequalities hold such that

$$
\rho\left[x_{n}, \xi\right] \geq \lambda^{n} \rho\left[x_{0}, T\left(x_{0}\right)\right]+b\left(1-\lambda^{n}\right)
$$

for every $n \in \mathbb{N}$.

Proof. Let $\xi, \eta \in X$ be such that $\xi=T \xi$ and $\eta=T \eta$. Then

$$
\rho[\xi, \eta]=\rho[T \xi, T \eta] \geq \lambda \rho[\xi, \eta]+b(1-\lambda),
$$

thus $(1-\lambda) \rho[\xi, \eta] \geq b(1-\lambda)$. Since $1-\lambda>0$ we infer that $\rho[\xi, \eta] \geq b$, whence $\rho[\xi, \eta]=b$ and $\xi=\eta$.

Let $x_{0} \in X$. Set $x_{1}=T\left(x_{0}\right), x_{2}=T\left(x_{1}\right), \ldots$; we are going to prove that $\left\{x_{n}\right\}_{n \in \mathbb{N}}$ tends to a limit $\xi$ and $T(\xi)=\xi$. The statement will thus be established. In this sense, we have

$$
\rho\left[x_{n+1}, x_{n}\right]=\rho\left[T\left(x_{n}\right), T\left(x_{n-1}\right)\right] \geq \lambda \rho\left[x_{n}, x_{n-1}\right]+b(1-\lambda),
$$

i.e., via the method of successive approximations, we obtain the following inequality

$$
\rho\left[x_{n+1}, x_{n}\right] \geq \lambda^{n} \rho\left[x_{1}, x_{0}\right]+b\left(1-\lambda^{n}\right) ;
$$

and thus, one deduces that if $m$ and $n$ are integers $\geq 0$, then

$$
\begin{gathered}
\rho\left[x_{n}, x_{m}\right] \geq \min \left\{\rho\left[x_{n}, x_{n+1}\right], \ldots, \rho\left[x_{m-1}, x_{m}\right]\right\} \geq \\
\geq \min \left\{\lambda^{n} \rho\left[x_{1}, x_{0}\right]+b\left(1-\lambda^{n}\right), \ldots, \lambda^{m-1} \rho\left[x_{1}, x_{0}\right]+b\left(1-\lambda^{m-1}\right)\right\}= \\
=\lambda^{n} \rho\left[x_{1}, x_{0}\right]+b\left(1-\lambda^{n}\right),
\end{gathered}
$$

i.e., $\rho\left[x_{n}, x_{m}\right] \rightarrow b(n \rightarrow \infty)$. This implies, by lower completeness, that the transversal sequence $\left\{T^{n}\left(x_{0}\right)\right\}_{n \in \mathbb{N}}$ converges, i.e., there is a $\xi \in X$ such that $T^{n}\left(x_{0}\right) \rightarrow \xi(n \rightarrow \infty)$. On the other hand, we have

$$
\rho\left[x_{n+1}, T(\xi)\right]=\rho\left[T\left(x_{n}\right), T(\xi)\right] \geq \lambda \rho\left[x_{n}, \xi\right]+b(1-\lambda),
$$

i.e., $\rho\left[x_{n+1}, T(\xi)\right] \rightarrow \lambda b+b(1-\lambda)$ as $n \rightarrow \infty$ or which is equivalently $x_{n+1} \rightarrow$ $T(\xi)$ as $n \rightarrow \infty$, i.e., from the preceding facts $\xi=T(\xi)$ and from (5) as $m \rightarrow \infty$ we obtain the inequality (Pr). The proof is complete. 
A brief proof of this statement may be found in Tasković [7].

Let $\left(X, \rho_{X}\right)$ and $\left(Y, \rho_{Y}\right)$ be two lower edges transversal spaces and let $T: X \rightarrow Y$.

In order, we notice from Tasković [7], that $T$ be lower edges continuous at $x_{0} \in X$ iff for every $\varepsilon>0$ there exists a $\delta>0$ such that the relation

$$
\rho_{X}\left[x_{0}, x\right]>b-\delta \quad \text { implies } \rho_{Y}\left[T\left(x_{0}\right), T(x)\right]>b-\varepsilon .
$$

A typical first example of a lower edges continuous mapping is the lower edges contraction on the lower edges transversal space $(X, \rho)$. For the further facts on the lower edges continuous mappings see: Tasković [7].

Theorem 3.4. Let $T$ be a mapping of a lower edges transversal space $(X, \rho)$ into itself and let $X$ be lower $T$-orbitally complete. Suppose that there exists a function $\varphi:[a, b] \rightarrow[a, b]$ satisfying

$$
(\forall t \in[a, b))\left(\varphi(t)>t \quad \text { and } \quad \liminf _{z \rightarrow t-0} \varphi(z)>t\right)
$$

such that

$$
\rho[T x, T y] \geq \varphi\left(\operatorname{diam}\left\{x, y, T x, T y, T^{2} x, T^{2} y, \ldots\right\}\right)
$$

for all $x, y \in X$. If $x \mapsto \operatorname{diam} \mathcal{O}(x)$ or $x \mapsto \rho[x, T x]$ is T-orbitally upper semicontinuous, then $T$ has a unique fixed point $\xi \in X$ and $\left\{T^{n}(x)\right\}_{n \in \mathbb{N}}$ converges to $\xi$ for every $x \in X$.

A brief proof of this statement may be found in: Tasković [7] with application of the following context.

Lemma 2. (Tasković [7]). Let the mapping $\varphi:[a, b] \rightarrow[a, b] \subset \mathbb{R}_{+}^{0}$ for $a<b$ have the property (Il). If the sequence $\left(x_{n}\right)$ of nonnegative real numbers satisfies the condition

$$
x_{n+1} \geq \varphi\left(x_{n}\right), \quad n \in \mathbb{N},
$$

then the sequence $\left(x_{n}\right)$ tends to $b$. The velocity of this convergence is not nesessarily geometrical.

A brief first proof of this statement may be found in: Tasković [7]. We notice that Lemma 2 is a dually form of Lemma 1.

As immediate consequences of the preceding Theorem 4 we obtain directly the following interesting cases of $(\mathrm{D})$ :

(6) There exists a nondecreasing function $\varphi:[a, b] \rightarrow[a, b] \subset \mathbb{R}_{+}^{0}$ for $a<b$ satisfying $\liminf _{z \rightarrow t-0} \varphi(z)>t$ for every $t \in[a, b)$ such that

$$
\rho[T x, T y] \geq \varphi(\operatorname{diam}\{x, y, T x, T y\})
$$


for all $x, y \in X$.

(7) (Special affine case of condition (D) for $\varphi(t)=\alpha t+b(1-\alpha)$ ). There exists a constant $\alpha \in[0,1)$ such that for all $x, y \in X$ the following inequality holds

$$
\rho[T x, T y] \geq \alpha \operatorname{diam}\{x, y, T x, T y\}+b(1-\alpha),
$$

i.e., equivalently to

$$
\begin{gathered}
\rho[T x, T y] \geq \\
\geq \alpha \min \{\rho[x, y], \rho[x, T x], \rho[y, T y], \rho[x, T y], \rho[y, T x]\}+b(1-\alpha) .
\end{gathered}
$$

(8) There exists a nondecreasing function $\varphi:[a, b] \rightarrow[a, b] \subset \mathbb{R}_{+}^{0}$ for $a<b$ satisfying $\liminf _{z \rightarrow t-0} \varphi(z)>t$ for every $t \in[a, b)$ such that

$$
\rho[T x, T y] \geq \varphi\left(\operatorname{diam}\left\{x, y, T x, T y, \ldots, T^{k} x, T^{k} y\right\}\right)
$$

for an arbitrary fixed integer $k \geq 0$ and for all $x, y \in X$.

(9) There exists an increasing mapping $f:[a, b]^{5} \rightarrow[a, b] \subset \mathbb{R}_{+}^{0}$ for $a<b$ satisfying $\liminf _{z \rightarrow t-0} f(z, z, z, z, z)>t$ for every $t \in[a, b)$ such that

$$
\rho[T x, T y] \geq f(\rho[x, y], \rho[x, T x], \rho[y, T y], \rho[x, T y], \rho[y, T x])
$$

for all $x, y \in X$.

In connection with the preceding facts, we are now in a position to formulate a localization of Theorem 4 in the following form.

Theorem 3.5. Let $T$ be a mapping of a lower edges transversal space $(X, \rho)$ into itself and let $X$ be lower $T$-orbitally complete. Suppose that there exists a function $\varphi:[a, b] \rightarrow[a, b]$ satisfying (Il) such that

$$
\operatorname{diam}\left\{T x, T^{2} x, \ldots\right\} \geq \varphi\left(\operatorname{diam}\left\{x, T x, T^{2} x, \ldots\right\}\right)
$$

for every $x \in X$. If $x \mapsto \operatorname{diam} \mathcal{O}(x)$ or $x \mapsto \rho[x, T x]$ is T-orbitally upper semicontinuous, then $T$ has a fixed point.

Proof of this statement is a totally analogous with the proof of Theorem 4 , and thus we omit it.

\section{References}

[1] M. R. Tasković: A generalization of Banach's contraction principle, Publ. Inst. Math., 37 (1978), 179-191.

[2] M. R. Tasković: Some results in the fixed point theory-II, Publ. Inst. Math., 41 (1980), 249-258.

[3] M. R. Tasković: Some theorems on fixed point and its applications, Annal. Soc. Math. Polonae, Series I, Comm. Math., 24 (1984), 323-334. 
[4] M. R. Tasković: A monotone principle of fixed points, Proc. Amer. Math. Soc., 94 (1985), 427-432.

[5] M. R. Tasković: Some new principles in fixed point theory, Math. Japonica, 35 (1990), 645-666.

[6] M. R. Tasković: Nonlinear Functional Analysis, Second Book: Monographs Global Convex Analysis - General convexity, Variational methods and Optimization, Zavod za udžbenike i nastavna sredstva and Vojnoizdavački zavod, Beograd 2001, (in Serbian), 1223 pages.

[7] M. R. Tasković: Theory of transversal point, spaces and forks, Monographs of a new theory, Beograd 2003, (in Serbian), 1000 pages, to appear.

[8] M. R. Tasković: Transversal spaces, Mathematica Moravica, 2 (1998), 133-142.

[9] M. Ohta and G. Nikaido: Remarks on fixed point theorems in complete metric spaces, Math. Japonica, 39 (1994), 287-290.

Matematički fakultet 11000 Beograd, P.O. Box 550

Serbia \& Montenegro

Received January 7, 2002. 